\title{
NOVEDADES EN HUPERZIA BERNH. (LYCOPODIACEAE) DE COSTA RICA
}

\author{
Alexánder Fco. Rojas Alvarado \\ Jardín Botánico Lankester, apdo. 1031-7050, Cartago, Costa Rica. afrojasa@hotmail.com
}

\begin{abstract}
One New SP ECIES AND two NeW ReCords of Huperzia (Lycopodiaceae) from Costa Rica are included here. The new species, $H$. oellgaardii A.Rojas is compared to H.taxifolia (Sw.) Trevis. and differs by subdimorphic leaves (vs. monomorphic), sterile leaves $2.5-3.5 \mathrm{~mm}$ wide (vs. $1.5-2.5 \mathrm{~mm}$ wide), fertile leaves 1.5-3.0 mm wide [vs. 1.0-1.5 (-2.0) mm wide] and distribution (1800-2900 m vs. $600-2300 \mathrm{~m}) . H$. chiricana (Maxon) Holub was collected in Volcán Turrialba and H. lancifolia (Maxon) Holub in Jicotea of Turrialba and Cerro Pittier of Coto Brus, Costa Rica.
\end{abstract}

Resumen. Se describe una especie nueva y se amplía la distribución de dos especies más de Huperzia (Lycopodiaceae) en Costa Rica. La nueva especie H. oellgaardii A. Rojas se compara con H. taxifolia (Sw.) Trevis. y difiere por varias características que se detallan en la descripción de la especie. H. chiricana (Maxon) Holub y H. lancifolia (Maxon) Holub se registran de nuevas localidades de Costa Rica.

Pal abr as cl ave / Key Words: Pteridophyta, Lycopodiaceae, Huperzia, Costa Rica.

El género Huperzia Bernh. es cosmopolita, con aprox. 400 especies en el mundo (Øllgaard 1995). Aunque fue descrito en 1801, se mantuvo en la sinonimia de Lycopodium L. por mucho tiempo. Holub (1983) eleva el nombre Huperzia de nuevo a la categoría de género y realiza numerosas combinaciones. Por su parte, Øllgaard $(1987,1988,1992)$ publica seis nuevas combinaciones. El mismo autor publica dos especies nuevas y una combinación nueva en su investigación del género para Flora Mesoamericana (Øllgaard 1993). Øllgaard (1995) registra 34 especies de este género en Costa Rica, de las cuales tres son endémicas. En la presente investigación se describe un nuevo taxon y se amplía la distribución de dos especies más de Huperzia de Costa Rica, con base en el siguiente acervo bibliográfico de Lycopodiaceae de Mesoamérica y Sudamérica: Holub (1983), Lellinger (1989), Øllgaard (1987, 1988, 1992, 1993, 1995), Rojas (1996). Además, se revisaron los especímenes del Herbario Nacional de Costa Rica (CR) y del Instituto Nacional de Biodiversidad (INB).

Huperzia chiricana (Maxon) Holub, Folia Geobot. Phytotax. 20: 71. 1985. Lycopodium chiricanum Maxon, Contr. U.S. Natl. Herb. 17(2): 176. 1913. Tipo: Panamá, Maxon 5364 (US). (Fig. 1).

Distr ibución. Costa Rica y Panamá, 3100-3400 m. Hasta ahora sólo conocida del Volcán Turrialba, en la Cordillera Central de Costa Rica, y en el Volcán Chiriquí de Panamá.

Nuevas l ocal idades. COSTA RICA. Cartago: Volcán
Turrialba, dentro del cráter, 3200-3300 m, 16 jun 1993, K. Mehltreter 425 (CR); Santa Cruz, Parque Nacional Turrialba, Volcán Turrialba, cráter, $10^{\circ} 01^{\prime} 30^{\prime \prime} \mathrm{N}$, 8345’35”W, 3200 m, 29 mar 2004, A. Rojas et al. 5555 (CR, MO, K).

Difiere de $H$. reflexa (Lam.) Trevis. por plantas más pequeñas $[5-10 \mathrm{~cm}$ de alto vs. $10-30(-40) \mathrm{cm}]$, hojas de 0.8-1.5 $\mathrm{mm}$ de ancho [vs. 0.5-1.0 (-1.2) $\mathrm{mm}$ ], lanceoladas a lanceolado-oblongas (vs. linearsubuladas), subcoriáceas a coriáceas (vs. herbáceas a subcoriáceas), distantes aproximadamente $1 \mathrm{~mm}$ entre las series [vs. (0.7-) 1.5-3.0 mm distantes], con la base entera a lobulada (vs. largamente dentada a ciliada), y el ápice pálido (vs. del mismo color que la lámina), dientes con lumen blanco (vs. lumen transparente) y superficie abaxial rugosa a acanalada cerca de la vena (vs. lisa y convexa).

Huperzia lancifolia (Maxon) Holub, Folia Geobot. Pitotas. 20: 74. 1985. Lycopodium lancifolium Maxon, Contr. U.S. Natl. Herb. 17(2): 177. 1913. Holotipo: Panamá, Maxon 5627 (US). (Fig. 2).

Dist r ibución: Costa Rica y Panamá, 1600-1900 m.

Nuevas local idades. COSTA RICA. Cartago: Turrialba, Tayutic, Jicotea, entre Río Jicotea y Río Vereh, $9^{\circ} 45^{\prime} 55^{\prime \prime} \mathrm{N}, 83^{\circ} 34^{\prime} 10^{\prime \prime} \mathrm{W}, 1800 \mathrm{~m}, 15$ jun 1995, G. Herrera \& I. Martínez 7894 (CR). Puntarenas: Coto Brus, Parque Internacional La Amistad, Cordillera de Talamanca, Estación Pittier, Sendero Altamira, 
9०01'30”N, 8257'40”W, 1700 m, 28 ene 1995, $A$. Mora et al. 16 (CR, INB).

Aunque esta especie fue citada por Lellinger (1988) del Alto de La Palma en San José, Øllgaard (1995) no la menciona de Costa Rica. Por tal motivo se informa de nuevo aquí.

\section{Huperzia oellgaardii A. Rojas, sp. nova (Fig. 3)}

Tipo: COSTA RICA. San José: Dota, along Río Pedregoso, $c a$. 1-2 km (by road) SE of Copey, 9³8'30”'N, 835'00”'W, 1940 m, 27 Febr 1990, M. Grayum et al. 9704 (Holotipo: CR, isotipo: MO).

A Huperzia taxifolia similis, sed foliis latioribus, sporangiis majoribus dignocenda.

Epífita de 25-40 cm de largo, decumbente, 1-4 veces dicotómicamente ramificada. Vástagos semi-heterofilos, en la base de 12-25 $\mathrm{mm}$ de diámetro (incluyendo las hojas), las regiones esporangiales terminales en los tallos, 7-15 $\mathrm{mm}$ de diámetro (incluyendo las hojas), a menudo en zonas diferenciables. Rizoma de 1.5$2.5 \mathrm{~mm}$ de grosor en la base (excluyendo las hojas), adelgazándose hasta 1-2 mm hacia arriba. Hojas basales de 15-25 x 2.5-3.5 mm, angostamente lanceoladas, ápice agudo, margen entero y plano, superficie lisa, ascendentes a casi adpresas, dispuestas en verticilos de 6-8 o en espiral, distantes $2-4 \mathrm{~mm}$ de la siguiente serie, adheridas en forma recta o a menudo oblicua, no retorcidas en la base, anchamente decurrentes, en la superficie adaxial ligeramente convexas a un poco cóncavas o canaliculadas, vena central débilmente prominente en el envés. Esporofilos de 5-15 x 1.5-3 $\mathrm{mm}$, ovados a lanceolados, generalmente ensanchados en la base, el ápice agudo, región apical poco convexa, acanalada o hasta abollada adaxialmente. Esporangios de 2.0-3.0 $\mathrm{mm}$ de ancho.

Distribución. Hasta ahora sólo conocida de la vertiente pacífica de la Cordillera Central y la Cordillera de Talamanca en Costa Rica, 1800-2900 m.

Paratipos: COSTA RICA. Cartago: Papales de San Juan, au flane sud de l'Irazú, 2900 m, 30 mar 1888, $H$. Pittier 151 (CR). Heredia: Sacramento, Montaña La Isla, $10^{\circ} 06^{\prime} \mathrm{N}, 84^{\circ} 07^{\prime} \mathrm{W}, 2300-2500 \mathrm{~m}, 13$ ene $1987, J$. Gómez 11308 (CR). Puntarenas: Buenos Aires, Ujarrás, cuenca superior del Río Ceibo, 9²1'10'N, 83¹6'38'W, 2200-2600 m, 5 ago 1989, M. Valerio 177 (CR), M. Valerio 184 (CR). San José: Santa Rosa de Copey, 1800 m, abr 1898, A. Tonduz 12234 (CR, AAU)

Huperzia oellgaardii difiere de H. taxifolia (Sw.) Trevis. por tallos semi-heterofilos (vs. comúnmente homofilos), hojas estériles de 2.5-3.5 $\mathrm{mm}$ de ancho (vs. 1.5-2.5 mm de ancho), esporofilos de 1.5-3.0 mm de ancho [vs. 1.0-1.5 (-2.0) $\mathrm{mm}$ de ancho], esporangios de 2-3 $\mathrm{mm}$ de ancho (vs. 1-2 $\mathrm{mm}$ de ancho) y por habitar a $1800-2900 \mathrm{~m}$ (vs. 600-2300 m). Por las hojas medianamente heterofilas, $H$. oellgaardii se observa como si fuese un estado intermedio entre $H$. taxifolia (prácticamente homofilas) y H. tubulosa (Maxon) B.Øllg. (marcadamente heterofilas), pero difiere de ambas por las hojas más desarrolladas. Probablemente esté más emparentada con $H$. tubulosa.

Øllgaard (1995) citó los especímenes Standley 42296 (US) y Tonduz 12234 (en parte, AAU) como una forma muy grande de Huperzia taxifolia (Sw.) Trevis., que probablemente ameritaba reconocimiento taxonómico. Por ello y por las múltiples contribuciones pteridológicas de este autor, se le dedica la nueva especie.

Agradecimientos. Agradezco al personal de los herbarios consultados, por permitirme utilizar sus colecciones, a Carlos O. Morales por la corrección del latín y a los revisores anónimos por sus comentarios sobre este artículo.

Lit er at ur a citada

Holub, J. 1983. Validation of generic names in Lycopodiaceae: with a description of a new genus Pseudolycopodiella. Folia Geobot. Phytotax. 18(4): 439-442.

Lellinger, D.B. 1989. The ferns and fern-allies from Costa Rica, Panama, and the Chocó. Part I. Pteridologia 2A. p. 24-43.

Øllgaard, B. 1987. A revised classification of the Lycopodiaceae. Opera Bot. 92: 153-178.

Øllgaard, B. 1988. Lycopodiaceae. In: Harling, G. \& L. Anderson (eds.). Flora of Ecuador 33: 1-155.

Øllgaard, B. 1992. Neotropical Lycopodiaceae - an overview. Ann. Missouri Bot. Gard. 79: 687-717.

Øllgaard, B. 1993. Two new Mesoamerican species and a new combination in Huperzia (Lycopodiaceae). Novon 3 (1): 67-72.

Øllgaard, B. 1995. Lycopodiaceae. In: Moran, R.C. \& R. Riba (eds.). Flora Mesoamericana. Vol. 1. Psilotaceae a Salviniaceae. Univ. Nac. Autónoma de México. p. 5-22.

Rojas, A.F. 1996. Aportes a la Flora Pteridophyta Costarricense. I. Informes. Brenesia 45-46: 1-6. 


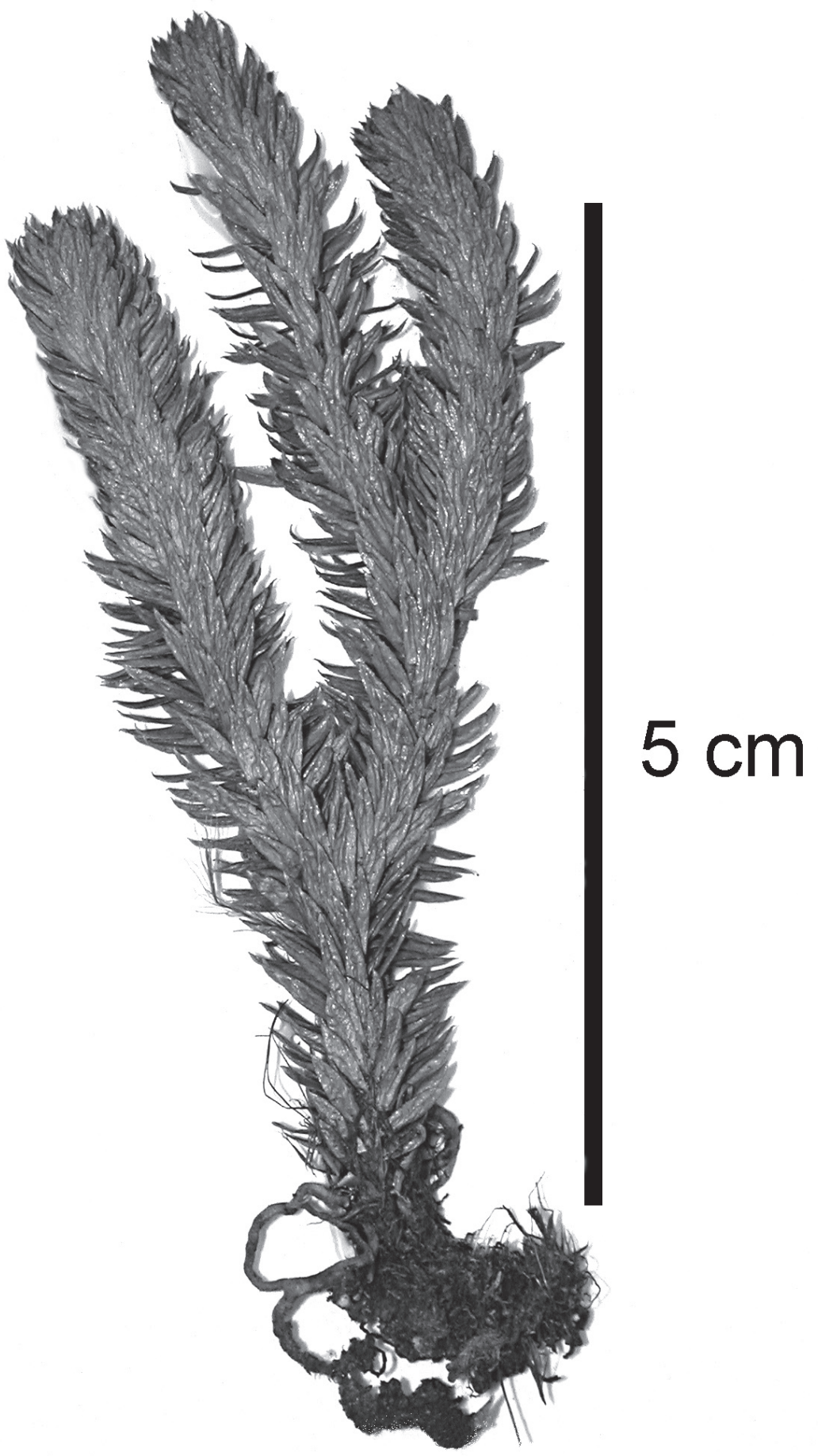

Fig. 1. Ejemplar representativo de Huperzia chiricana (Maxon) Holub (K. Mehltreter 425, CR). 

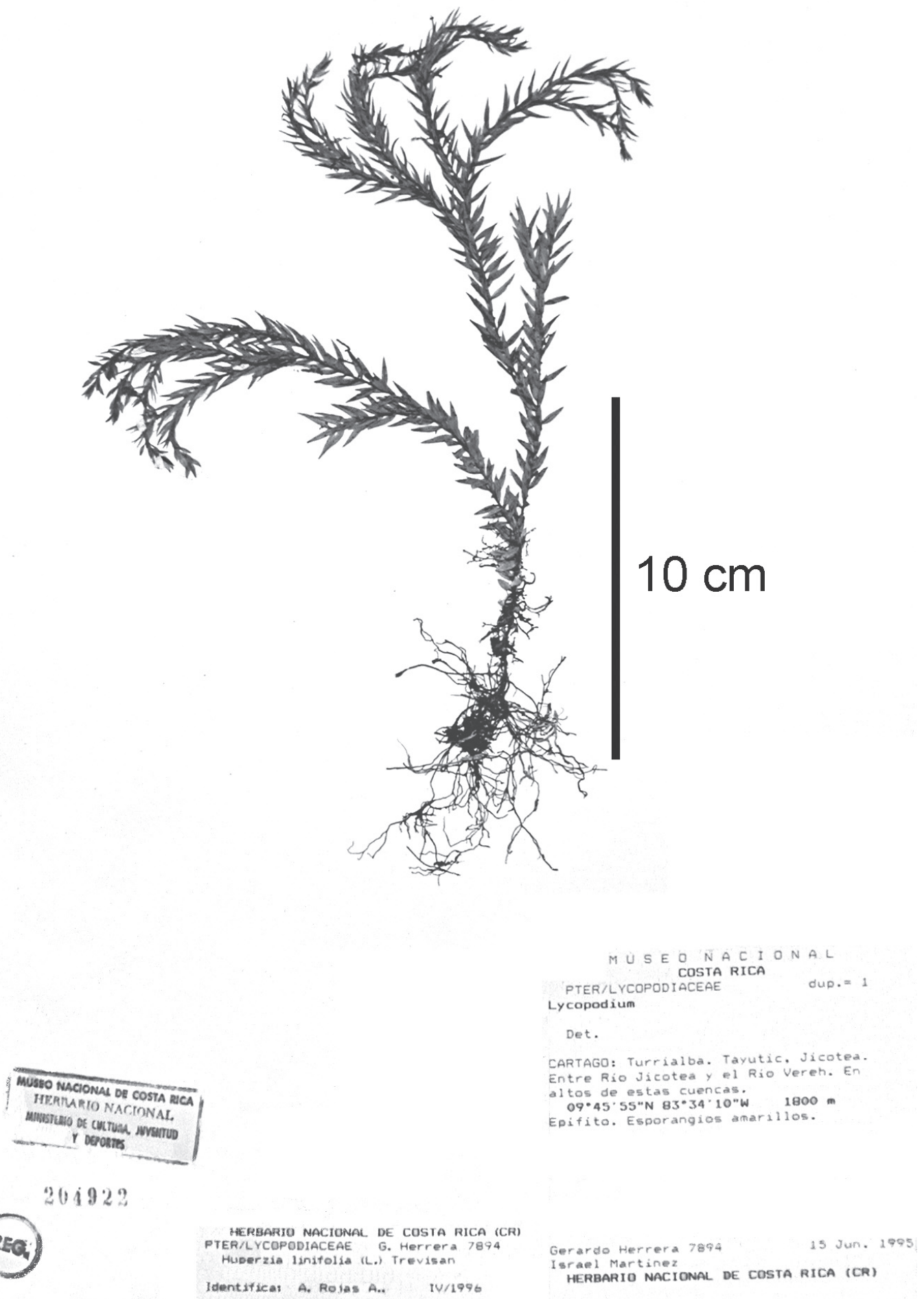

Fig. 2. Ejemplar representativo de Huperzia lancifolia (Maxon) Holub (G. Herrera \& I. Martínez 7894, CR). 


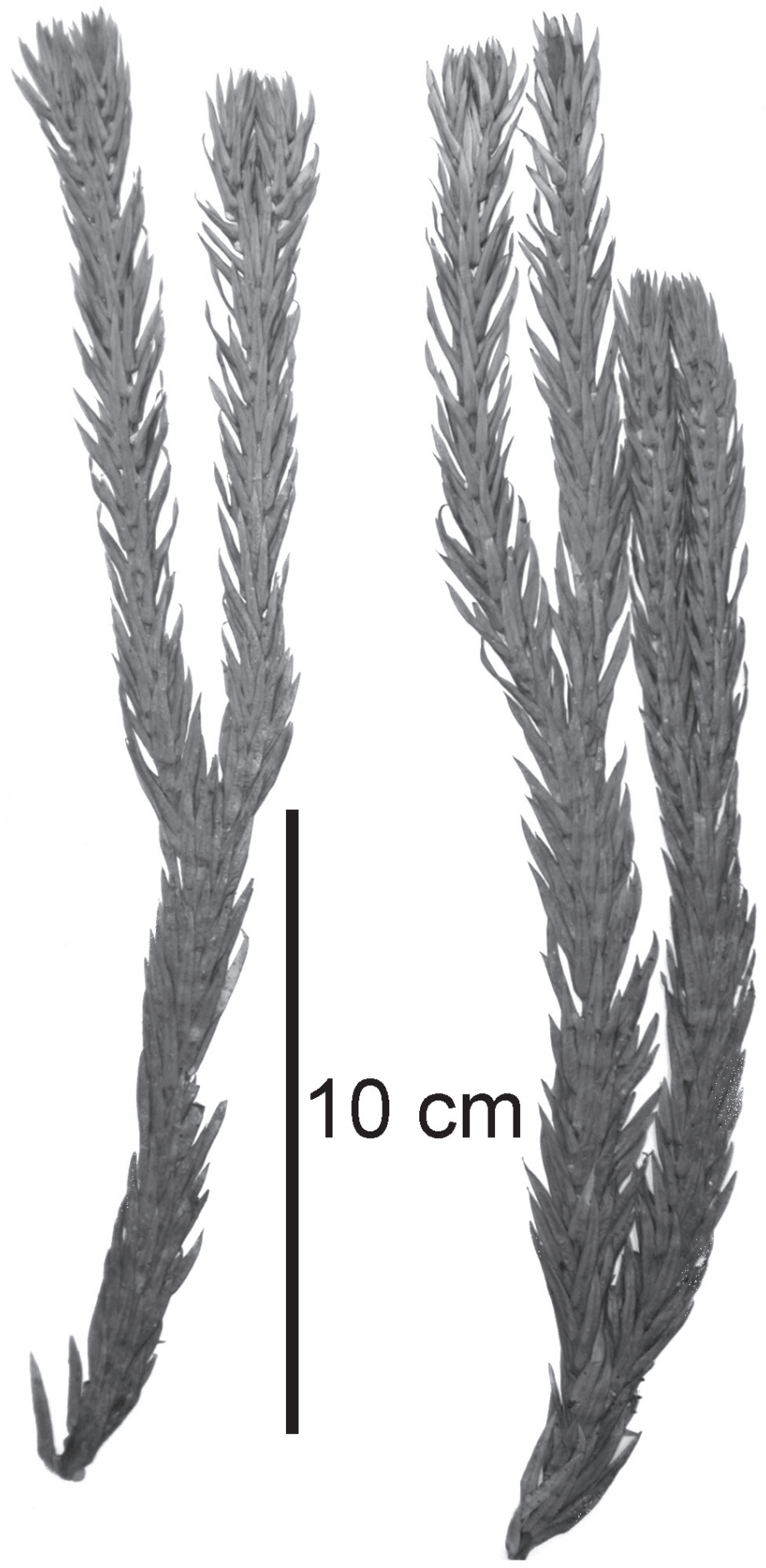

Fig. 3. Ejemplar tipo de Huperzia oellgaardii A. Rojas. (M. Grayum et al. 9704, CR). 
